"The final authenticated version of Polydorou, Doros, Ben-Tal, Oded, Damsma, Atser and Schlichting, Nadine (2020) VR: Time Machine in in: Masaaki Kurosu, editor (2020), 'Human-Computer Interaction. Human Values and Quality of Life' (LNCS volume 12183), ISBN 9783030490645, ISSN 0302-9743, pp. 294-306. is available online at https://doi.org/10.1007/978-3-030-49065-2_21 


\title{
VR:\ITime Machine
}

\author{
Doros Polydorou ${ }^{1}$, Oded Ben-Tal ${ }^{2}$, Atser Damsma ${ }^{3}$ and Nadine Schlichting ${ }^{3}$ \\ ${ }^{1}$ Cyprus University of Technology \\ ${ }^{2}$ Kingston University \\ ${ }^{3}$ University of Groningen
}

\begin{abstract}
Time Machine is an immersive Virtual Reality installation that explains - in simple terms - the Striatal Beat Frequency (SBF) model of time perception. The installation was created as a collaboration between neuroscientists within the field of time perception along with a team of digital designers and audio composers/engineers. This paper outlines the process, as well as the lessons learned, while designing the virtual reality experience that aims to simplify a complex idea to a novice audience. The authors describe in detail the process of creating the world, the user experience mechanics and the methods of placing information in the virtual place in order to enhance the learning experience. The work was showcased at the $4^{\text {th }}$ International Conference on Time Perspective, where the authors collected feedback from the audience. The paper concludes with a reflection on the work and some suggestions for the next iteration of the project.
\end{abstract}

\section{Introduction and background}

We can hear, smell, taste and feel our environment - together forming a rich perception of the world. Neuroscientists have a pretty good idea how these senses are processed in the brain. We evidently also have an inner sense of time. One modern theory of time perception suggest that we can perceive time based on neural oscillatory activity in the cortex. VR: \Time Machine is a virtual reality installation which explains the said neurobiologically plausible model of time perception. This work explores a number of different areas of investigations in the field of Virtual Reality. By using the immersive qualities of the medium, the experience builds upon the capabilities of the platform as a learning tool. Furthermore, it explores investigations of narrative structures in VR, using concepts such as localized sound sources, information placement in the $3 \mathrm{~d}$ space and interactivity as mechanisms to aid learning.

\section{Problem}

For the modern scientist, it has become increasingly important to communicate their work and findings to an audience other than their respective field of research [Greenwood \& Riordan, 2001]. While this is an important task, it is also a difficult one: One has to find the balance between simplification and scientific accuracy, identify scientific jargon and tell an engaging story. VR experiences offer the unique opportunity to explain science in a multisensory way - listening to a narration, seeing visualisations of the concepts being told, and being able to interact and actively explore the thing that is explained. This work aims to frame a theoretical model of how time is encoded in the brain into an easily understandable audio-visual and ludic experience.

\section{The SBF model}

Modern theories of time perception assume that the human brain can perceive temporal intervals, and therefore predict when a next instance of a regular event will happen (e.g., the next beep of an alarm clock), or whether a temporal interval will be long enough to perform a particular task within that interval (e.g., peek a quick look at a map while waiting at a red light). As we are able to estimate time even in the absence of any external information (e.g., in between two beeps of the alarm clock), the brain has to provide its own source of time. The brain consists of millions of neurons that communicate with each other through electrical signals. There are groups of neurons that fire these signals at a constant rate - like a regular drum beat. We call this phenomenon neural oscillations. Some groups oscillate faster than others, so that each group of neurons has its own unique speed. The striatal beat frequency (SBF) model proposes that when something interesting happens in the world, the oscillating neurons are reset and they synchronize with each other [Matell \& Meck, 2004]. Because of their difference in firing speeds they quickly drift out of synch again. This creates different patterns over time. So, at 
any particular moment this pattern can tell us how much time has passed. Recent work, based on neuroscience studies, suggests that neurons in the striatum, a central part of the brain, react to specific oscillatory activation patterns of cortical neurons, making them "time detectors" (see Fig. 1) [Buhusi \& Meck, 2005; Gu, Van Rijn \& Meck, 2015]. This way, we can predict events and anticipate actions, preventing us from looking at the map for too long.

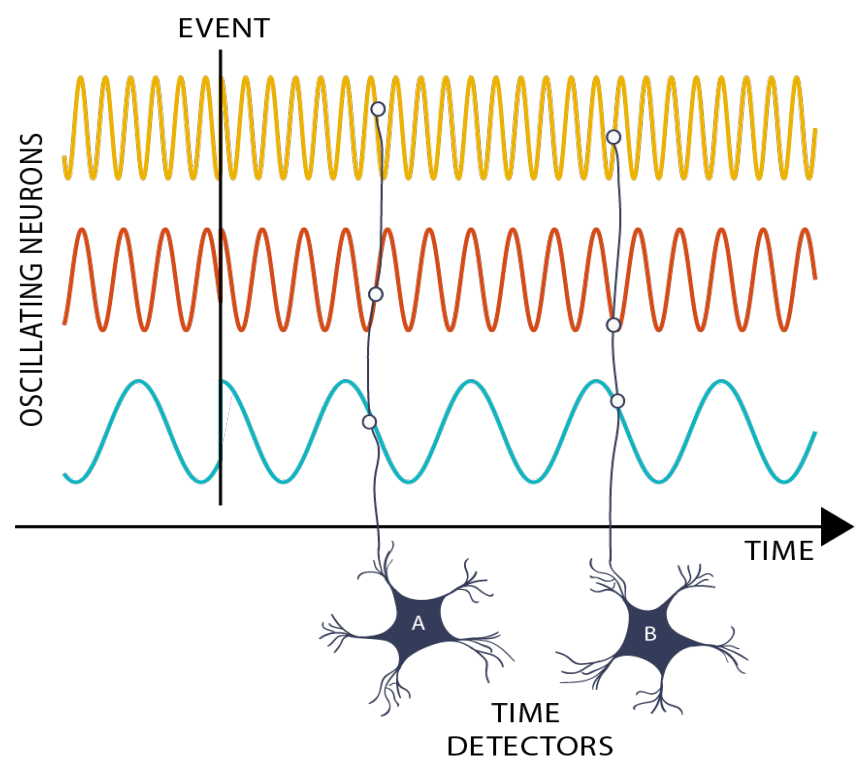

Fig. 1. Schematic depiction of the SBF model. Neurons oscillating at different speeds are synced or reset by an external or internal event. Time detector neurons located in the striatum react to different activation patterns that occur over time, allowing us to tell time.

Within the field of temporal cognition, the SBF model has been very influential because it aims to close the gap between theoretical psychological models and neuroscience. While the SBF model is not the last model to do so [see Merchant \& de Lafuente, 2014, for a review], it was one of the first.

The paper starts with a literature review on educational Virtual Reality experiences. It then continues by outlining the creation process, paying a special emphasis on the four immersion techniques followed by the team: Aesthetic choices, information placement, multi-mode experience, and audio. A first version of the experience has been presented on the 4th International Conference on Time Perspective, where the team manage to collect and reflect on audience feedback, which are outlined in the next section of the paper. Finally, in the concluding section, the authors identify certain elements of exploration for future work.

\section{Literature Review}

\section{Serious Gaming}

Science learning is generally considered to have an issue with engagement. Because the subject of science is often considered abstruse and challenging, many students actually cannot engage in science learning activities and fail to achieve better understanding of science (Lee \& Anderson, 1993). Student agency in simulated immersive environments is a credible way to allow students to experiment with science concepts and ideas even theoretical ones and many researchers have argued that, even as an entertainment medium, videogames support rich discursive and inquiry-based practices (for example, Gee, 2004, Steinkuehler, 2006; or Squire, 2006). Dede, talking about series games claims that it provides students with a subject impression of immersion that one feels like he/she is the avatar virtual world with a comprehensive, realistic experience, and research argues that immersion enhances science learning by at least authorizing multiple perspectives, situated learning and transfer (Dede, 2009). Furthermore, Barab et all argue that using video games in science education has made learner centered science learning more efficient and effective. In their view, serious gaming can facilitate student science learning through transformational play by situating the learner within a rich interactive context in which the scientific content is embedded in a series of authentic problems (Barab et al, 2009). Student 
motivation and engagement can be potentially increased because pleasure and instructional materials are combined into a whole in the virtual world, which allows the learner to engage in a recursive game cycle so that deeper learning is fostered (Squire, Barnett, Grant \& Higginbotham, 2004). Complex and abstract scientific concepts are visualized through tangible representations in the simulated game world, wherein one can generate hypotheses and test strategies iteratively without any need to worry about real-life consequences (Spires, Rowe, Mott \& Lester, 2011). Many natural phenomena - or in the context of this work, theoretical models - that cannot be produced in real-world situations, as well as many experiments and human behaviors that cannot be easily investigated, are allowed to be harmlessly simulated and evaluated in the game (Farrington, 2011; Kobes, Helsloot, de Vries \& Post, 2010).

Immersion

According to Brown and Cairns (2004), game immersion consists of three stages, with different barriers existing between the stages, such that a gamer cannot progress from one stage to the next until certain barriers are overcome. To get into the first stage, engagement, players must be satisfied with the game features and feel control over the game, as well as be willing to invest time and effort into the game. As players become further involved with the game, they enter the second stage, engrossment, in which their perceptions of their surroundings and physical needs decrease and their emotions are highly attached to the game. Finally, during the last stage, total immersion, individuals might feel like they are actually the avatars and thus empathize with their situations. When they have reached the stage of total immersion, players are entirely cut off from reality, and the game is all that matters to them. They are so absorbed in the game even to the extent as being in the game. However, total immersion is an intense experience that is relatively difficult to achieve.

Cheng et all conducted a number of studies to investigate how game immersion relates to learning. In their 2015 study, they employed construct validity approaches including exploratory and confirmatory factor analyses to confirm the three stages of game immersion experience and further verify its hierarchical structure. While all three stages of game immersion were positively correlated with student gaming performance (game scores, how students performed in the game) only the first stage, engagement, was positively correlated with students science learning outcomes (test scores, how students performed on science knowledge assessment).

Furthermore, their results indicated that gaming performance partially mediates the effects of game immersion experiences on science learning through serious gaming. Furthermore, their cluster analysis results demonstrated that two core clusters presented meaningful patters: high gaming performance / high immersion and high science learning / low immersion (Cheng, She \& Annetta, 2015).

\section{Sonification}

Just as data can be visualised to aid understanding, it can also be mapped to audio or sonified. Sonification was utilised in a wide range of research fields including health (Cassidy 2004, Poveda 2017), astronomy (Tomlinson 2017), Sports (Hummel 2010, Du 2018), or economics (Ben-Tal 2004, Chabot 2017) to name just a few examples. Perhaps even more than the graphic representation of data, effective sonification benefits from a deeper understanding of sound and music. Arbitrary mapping of data to audio parameters can become confusing to the users (Du 2018) and, as observed by Papachristodoulou (2014) below, some of the choices regarding type of sounds have to be guided by aesthetics to make the result engaging to the user.

Neuroscience research often involves very large and complex data-sets that are difficult to navigate. Papachristodoulou (2014) combined visualisation and sonification within an immersive 3D environment to represent simplified brain activation patterns. They mapped aspects of the data - number of nodes and connections, strength of the connections, hemispheric location - to sound. They utilised two types of sounds: a sustained, drone-like sound and brief bursts which they use in a manner akin to granular synthesis (Roads 2001).The data was used to modulate time, pitch, loudness, and stereo placement. They found that adding this sonification to the visual presentation enhanced users' understanding of the data.

Beyond making complex data comprehensible, sonification can also be an interface between science and artistic practice. Schmele (2012) also applied sonification to neural activation patterns to sound but their ultimate aim was creative - the outcome was an immersive installation. The data, fMRI of people listening to sound-stimuli, was used as the basis for generating music. The first stage transformed the time-sequence of voxel activation into the frequency domain. The spectral peaks, expressed as frequencies in the hearing range, was treated as synthetic musical scales. In the next stage musical material composed within those scales were projected into a 
spatialisation system using virtual speaker location controlled by the fMRI data. Listeners were surrounded by rapidly changing patterns of sound derived from brain activation patterns. Unlike the previous example, the aim here was not to communicate specific knowledge about brain anatomy or function. Rather it engaged users through an illustration of the richness and complexity of information processing that underpin even mundane tasks such as listening to rhythmic patterns.

Another example illustrates the porous nature of science communication and creative endeavour in this field. Weinberg (2006) discusses two projects that allow participants to engage with neural activation patterns. The second iteration of their approach provided users with physical interfaces to activate neural pathways. The activation patterns in the network were visualised and sonified via an array of speakers. Similar to Shmele above our ability to perceive location through sound is a good match to the distributive pattern of activation in a spatial network. But Weinberg also observes that on its own the spatialisation did not communicate the activity in the network well enough. But at the same time, supplementing this with visual representation led many users to focus on that element at the expense of the sonic side.

\section{Development}

The development was made up of a number of different stages. Firstly, we decided that the experience was going to offer two different modes. The story mode and the game mode. Following on from there, we create a storyboard, a script and we had a voice actor record a narration for us. We then proceeded to build the world in Unity, identifying the assets we wanted to use and recording some motions through a kinect motion-capture system. Continuing on, we designed and prototyped the user experience in Unity for both story and game mode and did some basic user testing, In this section, we will look at the different stages of the development and analyze the main methods and considerations.

The aim of the project was to allow users to understand and experience the SBF model of time perception. To this end, we decided to let a narrator guide the user through the key components of the model in a simple way, while the narration is being enforced by visual and auditory experiences. The narration consisted of a short text explaining: 1) why time perception is something we all use in everyday life, as illustrated by an example, 2) that time perception is not easy to study, since there is no organ for time as there is for other senses, and 3) how the SBF model proposes that time perception could be achieved in the brain. In the process of developing this text, we aimed to make sure that the explanations were easily understandable for a broad audience of users. This was done by linking time perception to our everyday experience and by avoiding any technical terminology that could be unfamiliar to most users.

\section{Overview of the experience}

When entering the experience, the viewer gets immersed into a VR world inhabited by neurons. The installation is made up of two parts. In part one, the viewer is guided through a narrated set piece that outlines the theoretical model. In part two the viewer assumes a more active role and becomes a player, trying out the model through a carefully designed ludic experience.

The experience is running in a loop. When a user is initially immersed, she will materialize in an ancient stone platform in a world of old ruins. Five groups of neurons will surround the user, pulsing at their own natural speeds. The user would be free to move around, approach the neurons and study them from up-close if they so wished. Looking beyond the platform, the user can see a portal where the same five pulses, appear from a top view perspective showcasing different patterns with each passing moment. The neurons are pulsating at specific intervals and a top down view of the same neurons can be seen through a stone portal. Even though it is not entirely evident at the once, the user can quickly identify that they are actually the same neurons by paying close attention between the visual and the audio cues. This is a way to train to the user to identify how the neurons are the pulses are actualized in the experience. As the user looks at the controller in their hands, they notice a subtle notification that asks them to press a button for the story mode to commence.

As soon as the button is pressed, the environment turns dark and a narrator, made of particles, appears in the scene. As the narrator explains each step of the model, different areas in the environment light up and visual illustrations are placed in different areas around the platform for future reference. 
When the narration is finished, the environment returns back to its original state, completing the loop. The surroundings now make more sense and the user now has gained the ability to interact with certain elements of the scene. Interactions are performed by pressing the trigger button on the controllers.

\section{Aesthetic choices and world building}

As soon as the users put on the VR helmet, they can see around them a black starry night. In the background, they can identify statues and broken murals. Ruins have always been associated with the workings of time. As this association exists in many peoples mind, we have decided to use the aesthetics as the background to our installations. Continuing with the transient theme, the narrator takes the form of a stream of particles in order to avoid showings of gender or age. The minimal aesthetics are complemented by an elevated black and white grid floor that that sets the boundaries of an arena where the whole experience will take place. The experience is build with room scale in mind, therefore the world is populated 360 degrees around the arena.

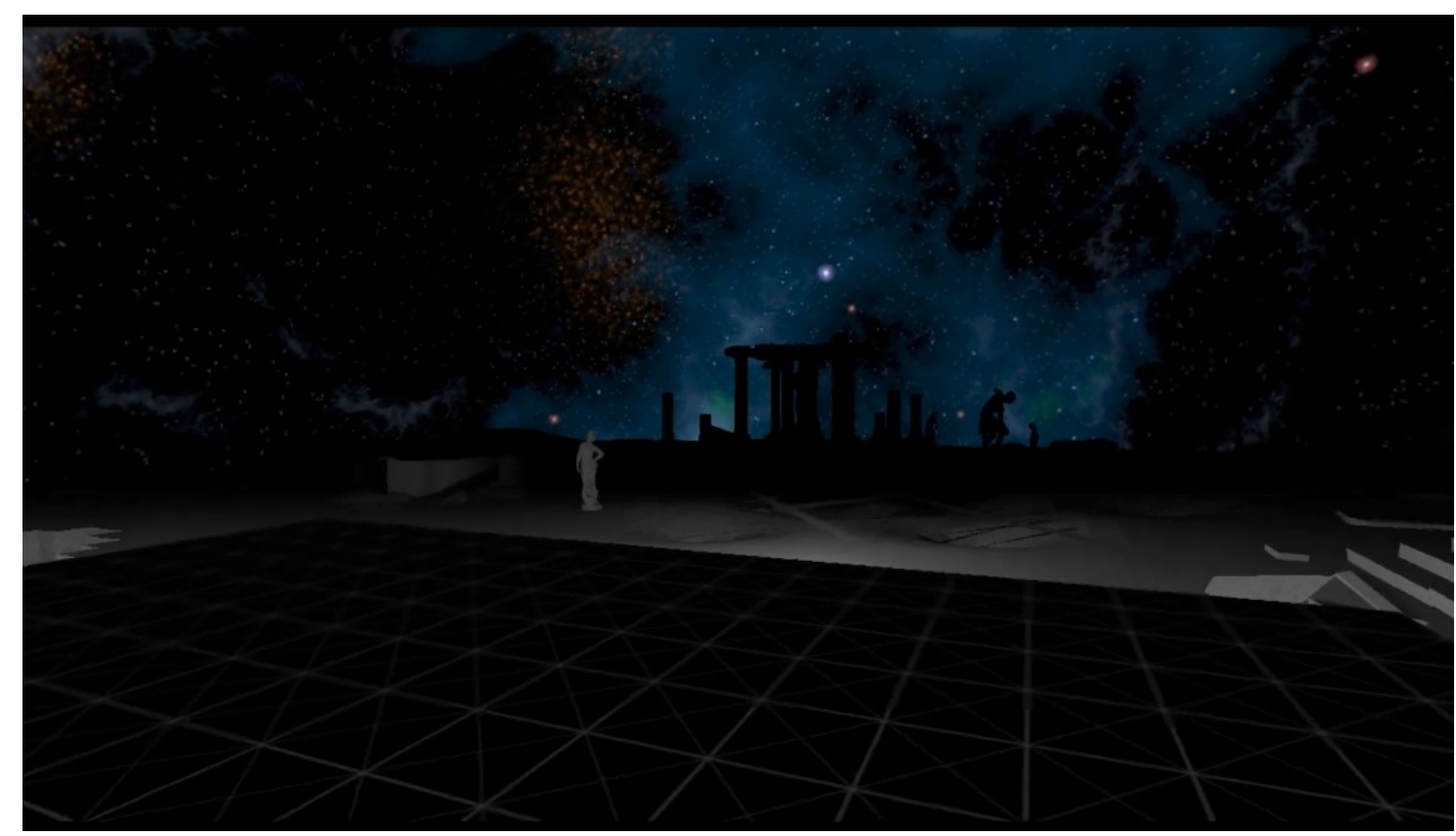

Fig 2 . The world as it is slowly unveiled to the users in story mode.

\section{Information placement}

One of the main challenges of story-telling in VR is how to attract and keep the attention of the user. For this experience, we decided to use the environment as a method of structuring information and controlling the attention of the viewer. The user is placed on an elevated platform in the center of the world and as the narrator is talking, information appears around the user in a fixed space. As more details are revealed about the model, the world gets more and more populated. By using spatial dynamics, we hope to simplify the internal computations needed to process the information. As the landscape is revealed, specific areas are "landmarked" through certain mechanisms. For example, a big group of pillars in placed in the background, or a distinct statue is revealed in a specific location. These specific landmarks were chosen because they are at the optimal visual proximity from the user. As the experience unfolds, the users identify those landmarks and uses them to create a mental image of the space in their heads. Information, such as the timings of pulsating neurons are then placed in front of those landmarks, making it easy for the user to make an association. 


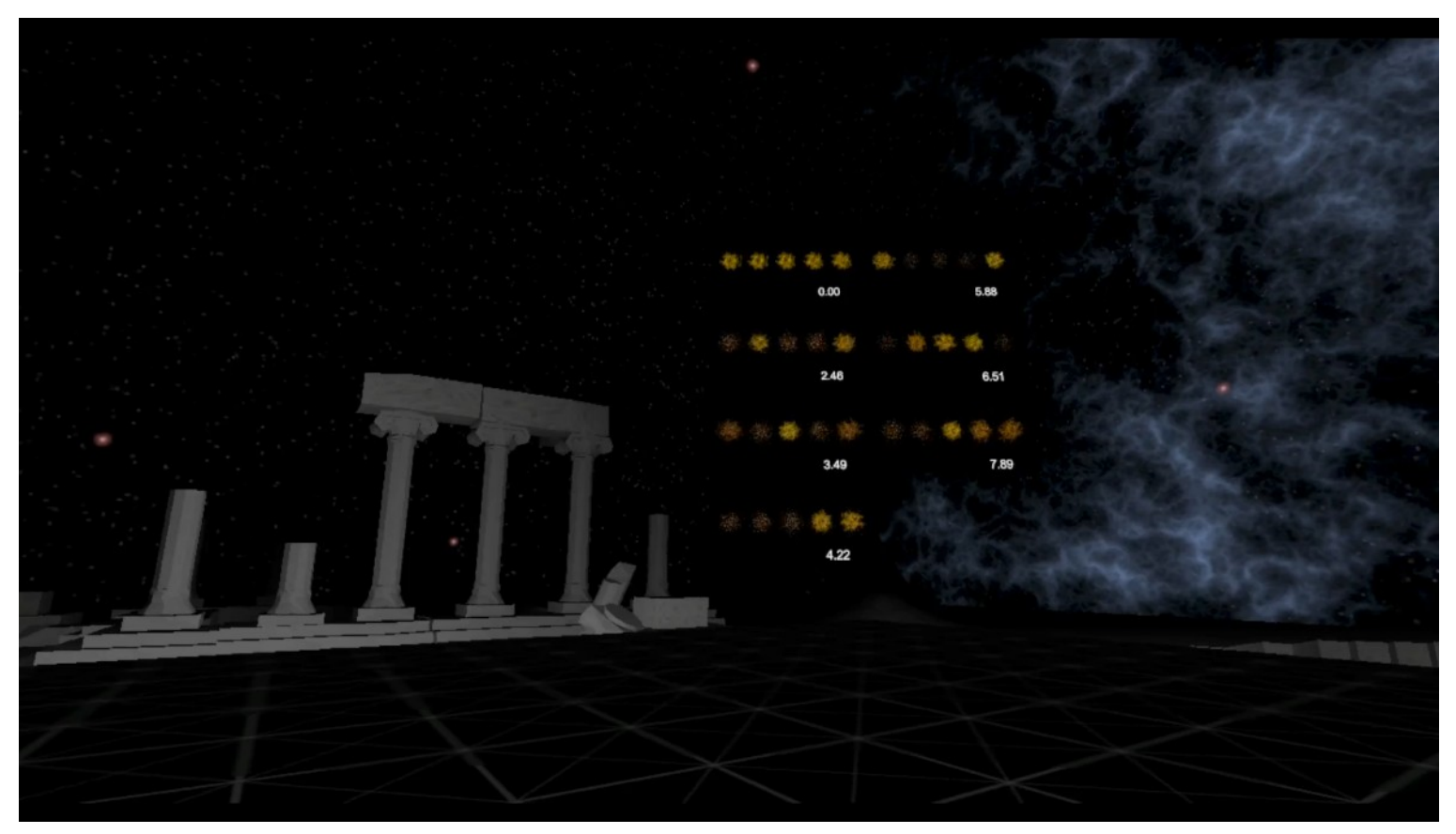

Fig3. Information placement around the space.

\section{Multi-mode experience}

The VR experience is split into two modes. The story mode and the play mode. The experience begins in the play mode, where the user can trigger changes in the environment. By pressing the story button, the world is cleared, the narrator explains the concept of the time perception model and at the end of the story mode the experience returns back to the play mode. We decided to take this approach as we didn't want to add a menu system to the experience, nor force the user to sit through the story every time. Furthermore, briefly interactive with the world both before and after hearing the explanation can aid users to piece the story together more efficiently.

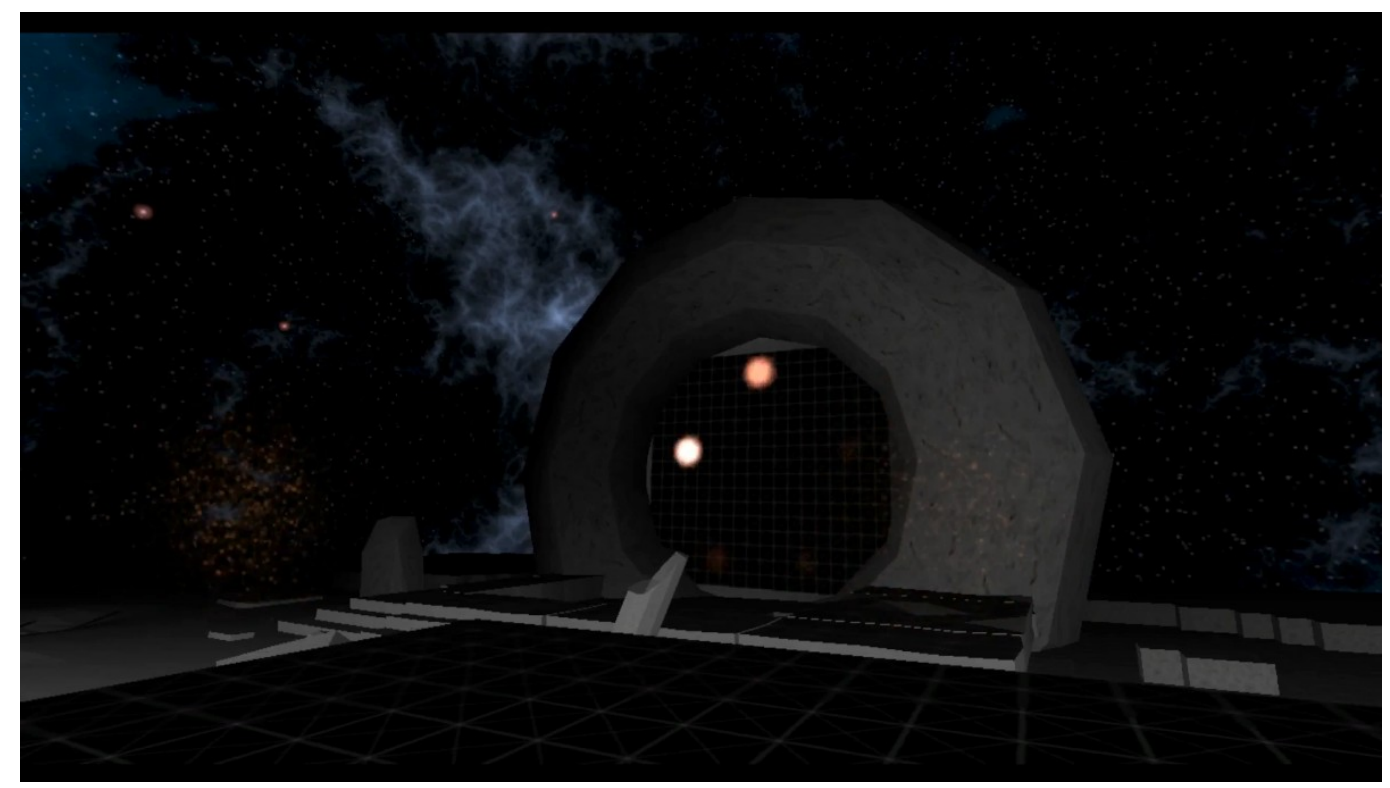

Fig4. Pulsating neurons in the play mode. 


\section{Sound}

We used three types of sounds in the work: (1) a speaker narrating the scientific explanation (2) sounds synchronised with the visual pulsation of the neurons ('sound effect') (3) Sustained background sounds that echoed the visual design of the scene ('atmospheric').

A central aspect of the model of time perception we are illustrating is the phase patterns between different groups of neurons each group pulsating at a regular speed. This opened a simple and direct way of sonifying this effect. Though in our case we are working on a computational model not measurements of neural activity. While the model suggests a very large number of pulsed neurons are involved we simplify things and illustrate the principle using just five. Short pitched sounds, with different pitch for each neuron, are synchronised with the regular oscillation of that neuron. By linking each of these pulse rates to a different sounded pitch, time intervals are manifested as melodic patterns as well as visual patterns of firing neurons. Both are presented to the user to help illuminate how the combination of regular pulses can be used as an internal clock. The synthesised sound is based on the Karplus-Strong plucked string model (Karplus 1983). Both the pitch and the pulsation rate we chose through a process of experimentation. The changing relationship had to be visually and aurally clear as well as appealing, and work practically with the duration we expected users to engage with the work. The 'chord' we ended up using is not a standard major/minor chord of Western Tonal music but is a collection of five notes including microtonal pitches ${ }^{1}$.

The sustained background sounds were used for purely aesthetic purposes in order to enrich the user experience of the VR environment. These were generated using granular synthesis (Roads 2001) where a source sound, singing in this case, is chopped into very short grains - $30 \mathrm{~ms}$ in this instance. The grains are then recombined to synthesise a novel sound. Granular synthesis is a very flexible method of generating new sounds. In this instance we were looking for background material that will match the visual scene of classical ruins while not distract the users. The sounds we synthesised were drone-like, though the parameter settings of the granular synthesis created sustained sounds with vague rather than sharp pitch definition. The narration explained the science we were trying to communicate to a wide audience. We developed the VR installation first using a recording we made ourselves. But we were able to get a better quality recording from a colleague who had experience producing audiobooks.

Sound in this project, therefore, follows cinematic conventions with dialogue as the most important element, and other sounds there to support the narrative and provide background. In this first iteration the sound was also presented in simple stereo. We plan to integrate sonic elements into the environment in the future. Sounds attached to objects and events in the scene can direct users to explore the space more. The main development we see for this project is in the interactive phase and there too sound can play a more significant and interesting role.

\section{Audience feedback}

The first evaluations were collected through informal interviews during the 4th International Conference on Time Perspective (France, August 2018). Overall, users responded positively to the VR experience. They complimented the aesthetic qualities of the visuals, the use of the avatar, the accessibility of the model explanation and the interactivity ("The world looks amazing and very immersive", "The avatar is really cool and it should appear more often", "The visuals and the sound helped me to understand how the pulses are linked to time". There were also some suggestions for improvement. Notably, some users had missed the ball-throwing example at the start of the narration, because they were still exploring the VR world (" The throwing scene could maybe be moved closer to the user, so that the ball also comes closer to the user or maybe the ball can have a more attention-grabbing color"). In addition, some noted that the sound and visuals of the pulsating neurons were slightly out of sync and that there could be more salient visual and auditory feedback when the neurons are 'reset' by the user. ("The sound seems to lack the visuals and be a bit out of synch". Finally, it was noted that the avatar was appealing and could appear more often ("Would be good if the avatar took a more substantial role, like a guide to the whole experience").

\section{Conclusion and future work}

\footnotetext{
${ }^{1}$ Microtones are notes that fall between the regular semi-tones represented by the white and black keys of the standard piano keyboard.
} 
With the VR: $\ \backslash$ Time Machine project, we aimed to explain a scientific computational theory - the SBF model to a diverse audience in an interactive, simple and enjoyable way. Using VR, the components of the model could be seen, as well as heard, in an immersive $3 \mathrm{D}$ environment. These components could also be manipulated by the user to enhance their understanding of the function of a particular component in the model.

During this work the authors build a virtual world by taking careful considerations of the user experience in virtual reality. The world was constructed in an aesthetic style with visual connotations to the ethereality of time. This has helped with the immersion qualities of the space as it situated the users into the right frame of mind. Continuing from there, an avatar was introduced to act as a guide to the experience and point to the user where to look and pay their attention during the experience. The user was situated in the center of the experience, in an elevated position, in order to be able to easily follow the narration around the circular space. The world was unveiled step by step, so the user could slowly absorb the information and as the narration was played out, notes appeared around the space creating a virtual learning canvas for the user to refer to. The visuals were complemented with sounds which played a very prominent role. The audio narration was driving the experience - complemented by the virtual avatar that appeared in various instances - and other sounds were there to support the visuals and to provide background.

Based on the audience feedback, several changes will be implemented in a subsequent version of the VR experience. We plan to develop the sonic aspect of the work to further enhance user engagement. The first aspect is to make use of spatialisation capabilities of VR environment. As noted above spatial cues are one of the interesting contributions of sound in this setting. We plan to integrate sounds into the virtual environment to encourage users to explore the environment more. In addition we plan to develop the interactive part of the installation more and here, again, audio can play a more significant role. Our aim is to demonstrate how the sural mechanism we describe can be used to estimate short durations. For instance, users can try to compare the duration of two time intervals. By varying relevant parameters in the model they can experience how those contribute to the measurement of time. Another option would be to illustrate what happens when the model is malfunctioning, as we believe is the case with some neurological disorders, and how this affects the accuracy of time estimations. Furthermore, we plan to use the avatar in even more parts of the experience as it seems the audience identified with it and it helped them to navigate in the virtual space. One more planned addition is to utilize the VR controllers a bit more. Currently, they are used to start or reset the experience but we aim to user them to offer supplementary information about the content to the user. As the narrator explains, the main points could appear in the form of bullet points in a virtual "notepad" attached to the controller. This mechanism can work in parallel with the information placement in the world space and it would allow the user to review during the story mode - the main ideas of the model and afterwards - during interaction mode - have a reference to what each button on the controller does.

\section{Credits}

The theoretical model of time perception has been proposed by Neuroscientists Matthew S. Matell and Warren H. Meck [1] and it has been adapted by researchers Atser Damsma and Nadine Schlichting for this installation. The virtual environment has been created by Doros Polydorou. The sounds and the music have been composed by Oded Ben-Tal and the narration has been voiced by Nathan Ridley (Hermitage Works Studio).

\section{References}

Barab, S., \& Dede, C. (2007). Games and immersive participatory simulations for science education: An emerging type of curricula. Journal of Science Education and Technology, 16(1), 1-3.

Barab, S. A., Cherkes-Julkowski, M., Swenson, R., Garrett, S., Shaw, R. E., and Young, M. (1999). Principles of selforganization: Ecologizing the learner-facilitator system. The Journal of The Learning Sciences $8(3 \& 4)$ : 349-390.

Ben-Tal, Oded, and Jonathan Berger. "Creative aspects of sonification." Leonardo 37, no. 3 (2004): 229-233.

Brown, E. \& Cairns, P. (2004). A grounded investigation of game immersion (pp. 1297-1300). Vienna, Austria: ACM Press. CHI '04 extended abstracts on Human factors in computing systems. 
Buhusi, C.V., Meck, W.H.: What makes us tick? Functional and neural mechanisms of interval timing. Nature Reviews Neuroscience 6(10), 755-765 (2005).

Chabot, S, and Braasch, J. "High-density data sonification of stock market information in an immersive virtual environment." The Journal of the Acoustical Society of America 141, no. 5 (2017): 3512-3512.

Cheng, M.T., She, H. C., \& Annetta, L. A. (2015). Game immersion experience: its hierarchical structure and impact on game-based science learning. Journal of Computer Assisted Learning, 31, 3, 232-253. doi: $10.1111 /$ jcal.12066.

Cheng, M. T., Lin, Y. W., She, H. C., \& Kuo, P. C. (2017). Is immersion of any value? Whether, and to what extent, game immersion experience during serious gaming affects science learning. British Journal of Educational Technology, 48(2), 246-263.

Cassidy RJ, Berger J, Lee K, Maggioni M, Coifman RR (2004) Auditory display of hyperspectral colon tissue images using vocal synthesis models. In: Barrass S, Vickers P (eds) Proceedings of the 10th international conference on auditory display (ICAD2004), Sydney, Australia.

Du, M., Chou, J. K., Ma, C., Chandrasegaran, S., \& Ma, K. L. (2018, April). Exploring the Role of Sound in Augmenting Visualization to Enhance User Engagement. In 2018 IEEE Pacific Visualization Symposium (PacificVis) (pp. 225-229). IEEE.

Farrington, J. (2011). From the research: myths worth dispellingêSeriously, the game is up. Performance Improvement Quarterly, 24, 105-110.

Gee, J. P. (2004). Language, Learning, and Gaming. A Critique of Traditional Schooling, Routledge, NY.

Gu, B.-M., Van Rijn, H., Meck, W.H.: Oscillatory multiplexing of neural population codes for interval timing and working memory. Neuroscience and Biobehavioral Reviews 48, 160-185 (2015).

Hummel, J, Hermann, T, Frauenberger, C, and Stockman, T. "Interactive sonification of german wheel sports." In Proceedings of ISon 2010-Interactive Sonification Workshop: Human Interaction with Auditory Displays. 2010 .

Karplus, K, and Strong, A.. "Digital synthesis of plucked-string and drum timbres." Computer Music Journal 7, no. 2 (1983): 43-55.

Lesen, A. E., Rogan, A., \& Blum, M. J. (2016). Science communication through art: objectives, challenges, and outcomes. Trends in Ecology \& Evolution, 31(9), 657-660.

Matell, M.S., Meck, W.H.: Cortico-striatal circuits and interval timing: coincidence detection of oscillatory processes. Cognitive Brain Research 21, 139-170 (2004).

Merchant, H., de Lafuente, V.: Neurobiology of interval timing (Advances in Experimental Medicine and Biology, 829). Springer, New York (2014).

Papachristodoulou, P., Betella, A., \& Verschure, P. F. M. J. (2014, March). Sonification of large datasets in a 3D immersive environment: A neuroscience case study. In ACHI2014: The Seventh International Conference on Advances in Computer-Human Interactions (pp. 35-40).

Poveda, J,O'Sullivan, M. Popovici, E, and Temko, A. "Portable neonatal EEG monitoring and sonification on an Android device." In 2017 39th Annual International Conference of the IEEE Engineering in Medicine and Biology Society (EMBC), pp. 2018-2021. IEEE, 2017.

Roads, C. (2001). Microsound. Cambridge, MA: MIT Press.

Kobes, M., Helsloot, I., de Vries, B. \& Post, J. (2010). Exit choice, (pre-)movement time and (pre-)evacuation behaviour in hotel fire evacuation-Behavioural analysis and validation of the use of serious gaming in experimental research. Procedia Engineering, 3, 0, 37-51. doi: 10.1016/j.proeng.2010.07.006.

Schmele, T., \& Gomez, I. (2012). Exploring 3D audio for brain sonification. Proceedings of the 18th International Conference on Auditory Display, Atlanta, GA, USA, June 18-21, 2012 
Steinkuehler, C. A. (2006). Massively multiplayer online videogaming as participation in a Discourse. Mind, Culture, \& Activity 13(1): 38-52

Spires, H. A., Rowe, J. P., Mott, B.W. \& Lester, J. C. (2011). Problem solving and game-based learning : effect of middle grade students' hypothesis testing strategies on learning outcome. Journal of Educational Computing Research, 44, 4, 453-472.

Squire, K. (2006). From content to context: Videogames as designed experiences. Educational Researcher 35(8): 19-29.

Tomlinson, B. J., Winters, R.M, Latina, C. Bhat, S. Rane,M. and Walker, B. N. "Solar System Sonification: Exploring Earth and its Neighbors Through Sound." The 23rd International Conference on Auditory Display 2017.

Weinberg, G., \& Thatcher, T. (2006, June). Interactive sonification of neural activity. In Proceedings of the 2006 conference on New interfaces for musical expression (pp. 246-249). IRCAM-Centre Pompidou. 